\title{
Constraining a Possible Variation of G with Type Ia Supernovae
}

\author{
Jeremy Mould ${ }^{1,2}$ and Syed A. Uddin ${ }^{1, *}$ \\ ${ }^{1}$ Centre for Astrophysics \& Supercomputing, Swinburne University of Technology, 10 John Street, Hawthorn, 3122, Australia \\ ${ }^{2}$ Corresponding author. Email: jmould@groupwise.swin.edu.au
}

(Received October 28, 2013; ACCEPTED February 6, 2014)

\begin{abstract}
Astrophysical cosmology constrains the variation of Newton's Constant in a manner complementary to laboratory experiments, such as the celebrated lunar laser ranging campaign. Supernova cosmology is an example of the former and has attained campaign status, following planning by a Dark Energy Task Force in 2005. In this paper, we employ the full SNIa data set to the end of 2013 to set a limit on G variation. In our approach, we adopt the standard candle delineation of the redshift distance relation. We set an upper limit on its rate of change $|\dot{G} / G|$ of 0.1 parts per billion per year over 9 Gyrs. By contrast, lunar laser ranging tests variation of $G$ over the last few decades. Conversely, one may adopt the laboratory result as a prior and constrain the effect of variable $G$ in dark energy equation of state experiments to $\delta w<$ 0.02 . We also examine the parameterisation $G \sim 1+z$. Its short expansion age conflicts with the measured values of the expansion rate and the density in a flat Universe. In conclusion, supernova cosmology complements other experiments in limiting $\mathrm{G}$ variation. An important caveat is that it rests on the assumption that the same mass of ${ }^{56} \mathrm{Ni}$ is burned to create the standard candle regardless of redshift. These two quantities, $f$ and $G$, where $\mathrm{f}$ is the Chandrasekhar mass fraction burned, are degenerate. Constraining $f$ variation alone requires more understanding of the SNIa mechanism.
\end{abstract}

Keywords: stars: (supernovae): general - gravity - (cosmology): distance scale - stars: (white dwarfs)

\section{INTRODUCTION}

The Planck mass, $m_{P}=\sqrt{ } \hbar c / G$, is a fundamental quantity of stellar astrophysics. The Chandrasekhar mass to order unity is $m_{C}=m_{p}^{3} / m_{p}^{2}$, where $m_{p}$ is the proton mass. The maximum mass of a star is approximately the Chandrasekhar mass multiplied by the square of the ratio of radiation pressure to gas pressure (Eddington 1917). The minimum mass of a black hole is within order unity of the Planck mass. Variation of Newton's constant affects supernova cosmology via change in the Planck mass over cosmic time and was first considered by Gaztañaga et al. (2002). At that time there were $42 \mathrm{SNe}$ available; there are now 581 (Suzuki et al. 2012).

Uzan (2003), Narimani, Moss, \& Scott (2012), and Moss, Nariamni, \& Scott (2010) advise that constraining the constancy of dimensional quantities is perilous. Preferred quantities are, for example, the 'gravitational fine structure constant', $\alpha_{\mathrm{g}} \equiv G m_{\mathrm{p}}^{2} / \hbar c$. The lunar laser ranging experiment initiated by NASA's Apollo mission is an $\alpha_{\mathrm{g}}$ experiment, monitoring the specific potential energy of the Earth-Moon system $G m_{\mathrm{p}}^{2} / c t$, where $t$ is the time of flight of Earth launched photons. Measuring the luminosity distance of galaxies, $D_{L}$, from type Ia supernovae is an $\alpha_{\mathrm{g}}$ experiment, as $D_{L}^{2} \propto m_{C} / m_{p}$,

\footnotetext{
* CAASTRO, http://www.caastro.org
}

assuming a fixed fraction, $f$, of $m_{C}$ is turned into energy and stellar luminosities are calibrated by hydrogen burning stars. Specifically, to within a numerical constant of order unity, $m_{C} / m_{p}=\alpha^{-3 / 2}$.

Speculation about varying $G$ began with Dirac (1937), who noted that the ratio of the electrostatic and gravitational forces between an electron and a proton was of the same order as the number of times an electron orbits a proton in the age of the Universe. He conjectured that $\alpha_{\mathrm{g}}$ might decay as the inverse of cosmic time. This 20th century gravity problem (which is sometimes tackled anthropically) has been totally eclipsed in the last decade by the cosmological constant problem (Solà 2013). The contribution to the vacuum energy density of fluctuations in the gravitational field is larger than is observationally allowed by some 120 orders of magnitude. Instead, the vacuum energy density is of the same order of magnitude as the present mass density of the Universe. Although ongoing type Ia supernova observations indicate that the equation of state of Einstein's General Relativity is the best fit, this gross cosmological constant problem provides no comfort for constant $G$ orthodoxy.

Garcia-Berro et al. (2007) review astronomical measurements and constraints on the variability of fundamental constants generally. Garcia-Berro et al. (2006) fit a polynomial 
Table 1. Constraints on the rate of variation of the gravitational constant.

\begin{tabular}{|c|c|c|c|c|c|}
\hline $\begin{array}{l}\dot{G} / G \\
10^{-13} y r^{-1}\end{array}$ & Current epoch & & $\begin{array}{c}\dot{G} / G \\
10^{-13} y r^{-1}\end{array}$ & Cosmic time & \\
\hline $2 \pm 7$ & Lunar laser ranging & (1) & $0 \pm 4$ & Big bang nucleosynthesis & (2) \\
\hline $40 \pm 50$ & Binary pulsar & (3) & $-1.42+2.48-2.27$ & Planck + WMAP + BAO & (4) \\
\hline \multirow[t]{3}{*}{230} & PSR J0437-4715 & (7) & $0 \pm 16$ & Helioseismology & (5) \\
\hline & & & $-6 \pm 20$ & Neutron star mass & (6) \\
\hline & & & $-300,+730$ & This paper & \\
\hline
\end{tabular}

Notes : the uncertainties are $1 \sigma$ unless otherwise noted.

1: (Muller \& Biskupek 2007); 2: (Copi, Davis, \& Krauss 2004); 3: (Kaspi et al. 1994); 4: (Li et al. 2013).

5: (Guenther, Krauss \& Demarque 1998); 6: (Thorsett 1996); 7: (Verbiest et al. 2008).

$G(z)=G_{o}\left(1-0.01 z+0.3 z^{2}-0.17 z^{3}\right)$ to the SNIa data, suggestive of a $G$ larger in the past. Verbiest et al. (2008) measure orbital period rates of pulsars and set a limit of $|\dot{G} / G|=23 \times 10^{-12} y r^{-1}$. From white dwarf cooling, Garcia-Berro et al. (2011) derive an upper bound $\dot{G} / G=-1.8 \times 10^{-12} y r^{-1}$ and Corsico et al. (2013) find a white dwarf pulsation limit of $\dot{G} / G=-1.3 \times 10^{-10} y r^{-1}$. Tomaschitz (2010) considers a gravitational constant scaling linearly with the Hubble parameter, and fits the SNIa Hubble diagram and AGN source counts, concluding that further observational constraints are required.

Furthermore, the luminosity of degenerate carbon core supernovae is proportional to the mass of carbon burned to ${ }^{56} \mathrm{Ni}$. The precise mechanism, which powers a type Ia supernova explosion, is a matter of lively debate, and we do not know yet whether a detonation or a deflagration occurs. What is clear is the close correlation between the mass of nickel synthesised in the outburst and the luminosity. This is discussed in quantitative experiments by Gaztañaga et al. (2002). The type SNIa standard candle is thought to result from a high degree of regulation of this quantity, such as would be provided by approach to a physical limit, the Chandrasekhar mass. However, one may conjecture that the fraction of $m_{C}$, which is turned into energy, may also vary with $z$. Like variation of $G$ with $z$, this is also an issue for constraints on the equation of state of the Universe arising from supernova measurements.

\section{VARYING $G$}

There are two constraints on varying $G$, that which has been established from the lunar distance since 1969 (current epoch in Table 1) and astrophysical constraints acting over cosmic time, such as the ages of the oldest stars. According to the theories of $G$ variation reviewed by Faulkner (1976), $G$ may have been larger in the past and may be considered to follow a $t^{-1}$ decline to the current epoch. Williams and Dickey (2002) placed a $1 \sigma$ limit of $\dot{G} / G=1.1 \times 10^{-12}$ per year in recent time. If $G$ exceeded the present value by $-3>\dot{G} / G>+7.3 \times$ $10^{-11}$ per year $13.7 \mathrm{Gyrs}$ ago, and we assume the supernova luminosity scales with the Chandrasekhar mass, we obtain Figure 1. This includes variation of the density term in the Friedmann equation, i.e. $\Omega_{m}(\mathrm{t})$ with two cases (1) $\Omega_{m}(t)+$ $\Omega_{\Lambda}(t)=1$ to retain flatness and (2) the dark energy density
$\Omega_{\Lambda}=0.73$ with $\Omega_{k}=1-\Omega_{m}(t)-\Omega_{\Lambda}(t)$. We characterise type (2) models by the value of the curvature, $\Omega_{k}$, at $z=0.5$. We adopt the WMAP9 limits on curvature (Hinshaw et al. 2013), $-0.0065<\Omega_{k}<0.0012$.

\subsection{The supernova Ia constraint}

The current supernova data (Suzuki et al. 2012) are shown in Figure 1. If we assume $\Lambda \mathrm{CDM}$ cosmology with $w=-1$, current SNIa data constrain $G$ stability to $\dot{G} / G=(-3,+7.3) \times$ $10^{-11}$ per year. This constraint is obtained if we adopt the standard model of cosmology as a prior. We now (1) reexpress this as a constraint on $\dot{\alpha}_{\mathrm{g}} / \alpha_{\mathrm{g}}$, and (2) invert the argument to constrain $w$, given laboratory limits on $G$ variation.

(1) To determine the upper limit on $\dot{\alpha}_{\mathrm{g}} / \alpha_{\mathrm{g}}$, we calculate $\chi^{2}$ to compare the data with the prediction, marginalising over $\mathrm{H}_{0}$, and show this in Figure 2. The contours of $\chi^{2}$ are oriented close to vertical, resulting in clear limits on $G$ variation. This constraint, our main result, $-3<10^{11} \dot{G} / G<7.3$ per year, may be expected to strengthen towards parity with the laboratory, in the era of dark energy experiments such as LSST; see Weinberg et al. (2013). An equivalent dimensionless limit is $-0.5<\dot{G} /\left(G H_{0}\right)<1$, where $1 / H_{0}$ is the age of the Universe.

(2) This constraint is obtained if we adopt the standard model of cosmology as a prior. However, these SNIa data are conventionally used as a measurement of $\Omega_{\Lambda}$. There is therefore a degeneracy between this and $\dot{G}$ addressed by the same data. We can quantify the degeneracy using the generalisation of the Friedmann equation as a polynomial by Mould (2011). Mould showed that if, such a polynomial is adopted to fit Figure 1,

$$
\left(H / H_{0}\right)^{2}=\Sigma_{n}(1+z)^{n} \Omega_{n}=h^{2}(z),
$$

relationships (degeneracies) between the $\Omega_{n}$ coefficients result from the limited available constraints (SNIa, CMB). If the SNIa data are used to constrain the equation of state of the Universe with $\mathrm{w} \approx-1$, there is therefore a degeneracy between $w$ and $\dot{G}$ addressed by the same data. For $z \sim 1$ and zero curvature, $\delta w \approx 2 \delta \Omega_{m}$ from Equations (3) and (11) of Mould (2011). For $G$ stability to two parts in $10^{12}$ per year, $\delta \alpha_{\mathrm{g}} / \alpha_{\mathrm{g}}=$ 0.0137 at $z=1$, which corresponds to $\delta w \approx 0.03$. The current experimental uncertainty in $w$ (Rapetti et al. 2013) is 0.07 . Both quantities therefore need to be constrained jointly. On 
Luminosity distance/redshift -- dashed line is standard model
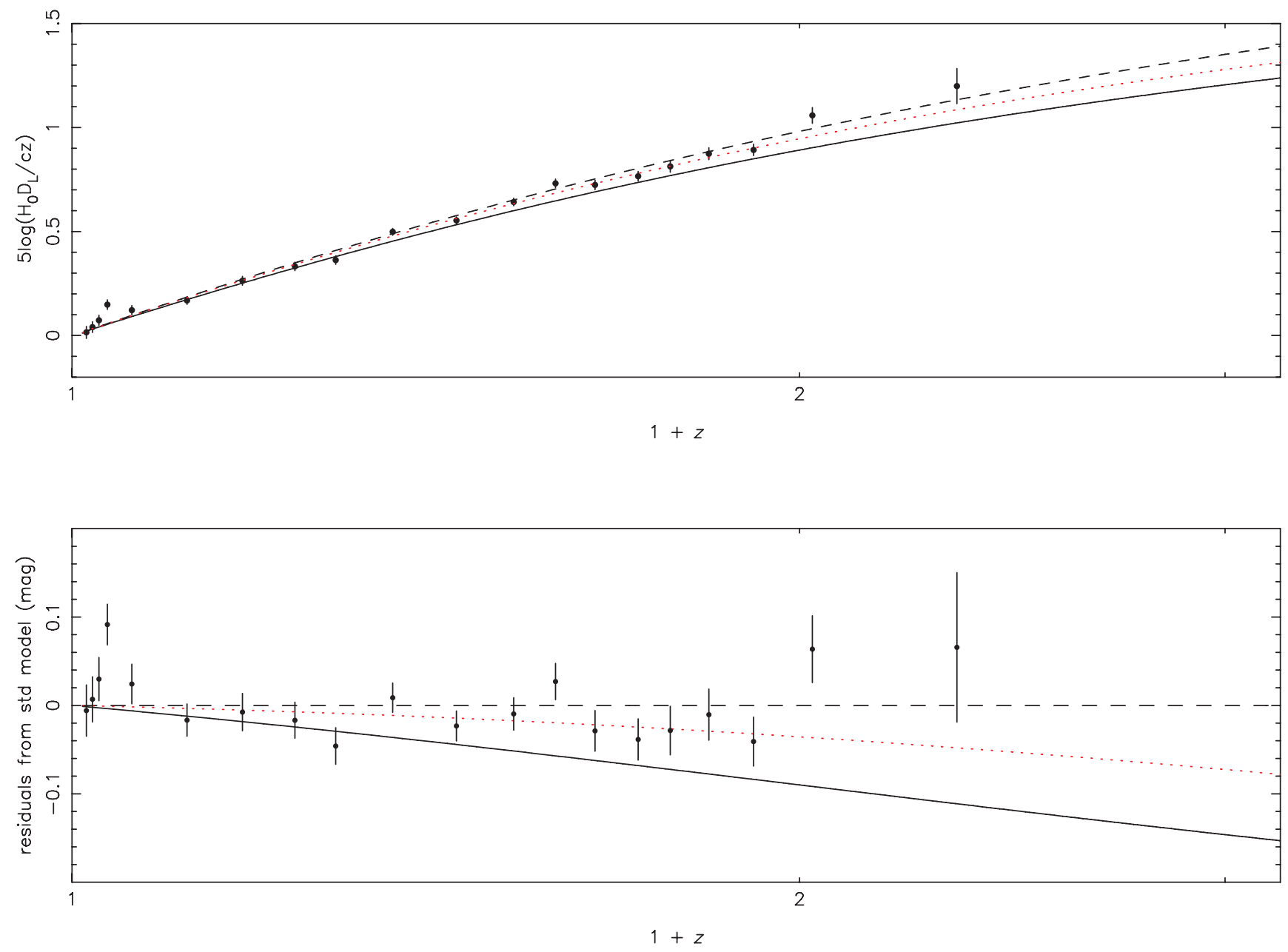

Figure 1. Luminosity distance versus redshift. The lower plot shows residuals from the standard model. The solid symbols are supernovae. The standard model is the dashed curve. The solid line and the red dotted line are the $G$ varied expectations, the former with non-zero curvature. The most distant SNIa is at 9 billion light years in the standard cosmology with $H_{0}=73.8 \pm 2.4 \mathrm{~km} / \mathrm{s} / \mathrm{Mpc}$, given by Riess et al. (2012).

the other hand, if one is prepared to adopt the lunar laser ranging results as a prior on dark energy experiments valid over all of cosmic time, the effect of variable $G$ is constrained so that $\delta w<0.02$ (95\% confidence). This is not a negligible contribution to the $w$ error budget, and it should not be ignored (cf. Mortonson et al. 2014).

Finally, the coupling of $D_{L}, f$, and $m_{C}$ is direct. Analytically, $2 \delta D_{L} / D_{L}=\delta f l f=-1.5 \delta G / G=\delta m_{C} / m_{C}$. Our limit on $\delta \alpha_{\mathrm{g}} / \alpha_{\mathrm{g}}$ is thus degenerate with an equivalent limit on $\delta f l f$.

\subsection{Other parameterisations}

Pragmatically, the key result here devolves from an assumed $t^{-1}$ variation of $\alpha_{\mathrm{g}}$. Its basis is historical and traces back to Dirac's (1937) large numbers hypothesis and the steady state Universe, neither of which have any real traction today. Other parameterisations are possible and even natural, such as $1+z$ scaling. One form is $\alpha_{\mathrm{g}}=\alpha_{0}+\alpha^{\prime} z$. In this case we obtain $-0.02<\alpha^{\prime} / \alpha_{0}<0.04$.

If $\mathrm{G} \sim 1+z$, the $\Omega_{3}$ coefficient in Equation (1) is promoted to $\Omega_{4}$; that is, it becomes an anti-radiation pressure term. Assuming $\Omega_{1}=0$, the resulting degeneracies can be expressed (Mould 2011)

$$
\left(f_{0}-f_{2}\right) \delta \Omega_{0}=\left(f_{2}-f_{4}\right) \delta \Omega_{4},
$$

where

$$
f_{n}=\int_{0}^{z}\left(1+z^{\prime}\right)^{n} h^{-3}\left(z^{\prime}\right) d z^{\prime}
$$

Any $G$ variation that scales as $1+z$ is traded off against $\Omega_{\Lambda}$, according to (from Table 2) $\delta \Omega_{\Lambda}=\delta \Omega_{0}=301.5 \delta \Omega_{4}$, when SNIa and CMB anisotropy data measure cosmological parameters simultaneously. A universe with just conventional dark energy and 'radiation' like this has an age in units of 


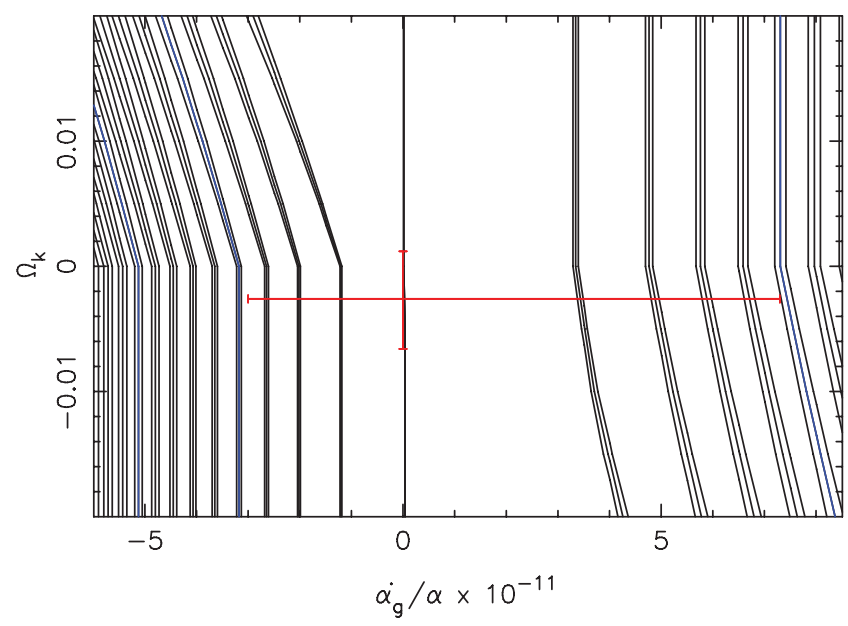

Figure 2. A vertical central valley of parameter space is permitted by these $\chi^{2}$ contours. The first contour on either side of zero is $\chi^{2}$ per degree of freedom $=1.2$ with a spacing between triplet contours of 0.2 . Positive values of $\dot{\alpha}_{\mathrm{g}}$ have the sense of $G$ larger in the past. With the WMAP9 limits on curvature (Hinshaw et al. 2013) shown by the vertical error bar this implies a SNIa cosmology constraint on G stability in the standard cosmology of $(-3,+7.3) \times 10^{-11}$ per year, evaluated at $\chi^{2}=2$.

Table 2. Equation of state components.

\begin{tabular}{llll}
\hline \hline$\Omega_{n}$ & $n$ & $\mathrm{w}_{n}$ & $f_{n}$ \\
\hline Vacuum & 0 & -1 & 0.662 \\
Textures & 1 & $-2 / 3$ & 0.964 \\
"Curvature" & 2 & $-1 / 3$ & 2.294 \\
Matter & 3 & 0 & 10.40 \\
Radiation & 4 & $+1 / 3$ & 494.4 \\
\hline
\end{tabular}

The $f_{n}$ coefficients have been evaluated at $\Omega_{3}=0.27$.

$1 / \mathrm{H}_{0}$ obtained by integrating Equation (1) with $a^{-1}=1+z$.

$$
\begin{gathered}
t=\int_{0}^{1} \frac{a d a}{\dot{a} a}=\int_{0}^{1} \frac{d a}{H_{0} a \sqrt{ }\left(\Sigma_{n} \Omega_{n} a^{-n}\right)} \\
t H_{0}=\int_{0}^{1} \frac{d a}{a \sqrt{ }\left(\Omega_{0}+\Omega_{m} a^{-4}\right)}=\int_{b}^{\infty} \frac{d y}{2 \sqrt{ } \Omega_{0} y \sqrt{ }\left(1+y^{2}\right)} \\
=\frac{1}{2 \sqrt{ } \Omega_{0}} \int \operatorname{cosech}(\mathrm{x}) d x=\frac{1}{2 \sqrt{ } \Omega_{0}}[\ln |\tanh (\mathrm{x} / 2)|]_{\operatorname{arsinh}(\mathrm{b})}^{\infty} \\
=\frac{1}{2 \sqrt{ } \Omega_{0}} \ln (\tanh (\operatorname{arsinh}(\mathrm{b}) / 2)),
\end{gathered}
$$

where

$$
b^{2}=\Omega_{m} / \Omega_{0}=\Omega_{m} /\left(1-\Omega_{m}\right)
$$

and

$$
y=b a^{-2}=\sinh (x) .
$$

For $\Omega_{M}=0.27, x=0.575$ at $z=0$ and the age is 0.745 . This is a second contradiction with the standard model of cosmology, as Planck finds an age approximately one in these units (Ade et al. 2013, Efstathiou 2013). A further contradiction with the age of the globular clusters is mildly ameliorated by higher central temperatures of stars $\left(G M m_{p} / k R\right.$, where
$M, R$ are the stellar mass and radius) during the epoch of re-ionisation, when they were formed, and the extraordinary temperature sensitivity of the $\mathrm{CN}$ cycle of fusion, but for most of the low mass stars' lifetime core temperatures are close to normal and ages are only mildly affected (Vandenberg 1977).

How severe a constraint on $G \sim 1+z$ is this? Error analysis gives terms in $\delta \Omega_{M} / \Omega_{M}, \delta \Omega_{\Lambda} / \Omega_{\Lambda}$ and $\delta H_{0} / H_{0}$. The first and last of these are of order a few percent and the second is smaller. This parameterisation can therefore be rejected with 99\% confidence. $G \sim(1+z)^{1 / n}$ would be less unacceptable for large $n$, but is not a natural parameterisation.

\section{SUMMARY}

Our conclusions from this work are as follows.

1. The validity of the SNIa standard candle depends on the stability of $G$ and the stability of $f$, the fraction of the Chandrasekhar mass turned into energy. We have considered the former in this paper and derived a constraint on the gravitational fine structure constant which can be compared with other combined astrophysicalcosmological constraints. But this is inextricably degenerate with possible evolution of $f$ due to changes over cosmic time of SNIa progenitor astrophysics. With this caveat, we set a SNIa cosmology constraint on $G$ stability in the standard cosmology of $(-3,+7.3) \times$ $10^{-11}$ per year.

2. This limit is two orders of magnitude weaker than that from lunar laser ranging. But that is a current epoch result and complements, but does not replace, a constraint that spans cosmic time.

3. The limit is also two orders of magnitude weaker than that arising from the great sensitivity to density of Big Bang nucleosynthesis. The SNIa standard candle, however, has the distinct advantage of covering the last $10^{10}$ years of cosmic time, rather than the first 20 minutes.

4. Our result is an update of Gaztañaga et al. (2002), who found $\dot{G} / G<12 \times 10^{-12} h_{70} /$ yr for $\Omega_{\Lambda}=0.8, \Omega_{M}=$ 0.2 . This is a $1 \sigma$ limit, like ours, and directly comparable since $h_{70} \approx 1$. The order of magnitude more supernovae now available have allowed us to relax their flat Universe assumption, but has also relaxed their limit on $\dot{G}$.

5. Caution would dictate that experiments to measure the equation of state of the Universe carry the caveat that $f$ and $G$ stability is assumed. For the latter, lunar laser ranging is available as a prior and limits $\delta w$ to 0.02 , but the former has not been quantified and demands further understanding of the SNIa mechanism.

6. A parameterisation $G \sim 1+z$ is interesting on theoretical grounds related to the unity of forces. However, with such an equation of state the expansion age of the Universe is too short. That parameterisation can therefore be rejected with $99 \%$ confidence. 


\section{ACKNOWLEDGEMENTS}

The authors are grateful to Chris Blake and Michael Murphy for helpful advice and to an anonymous referee for emphasising the issue of the quantum of ${ }^{56} \mathrm{Ni}$. CAASTRO is the ARC's Centre of Excellence for All-Sky Astrophysics, funded by grant CE11001020.

\section{REFERENCES}

Ade, P., et al. 2013, A\&A, in press, astro-ph 1303.5076

Copi, C., Davis, A., \& Krauss, L. 2004, PhRvL, 92, 171301

Corsico, A., et al. 2013, JCAP, 06, 032

Dirac, P. 1937, Natur, 139, 323

Eddington, A. S. 1917, MNRAS, 77, 596

Efstathiou, G. 2013, astro-ph 1311.3461

Faulkner, D. 1976, MNRAS, 176, 621

Garcia-Berro, E., et al. 2006, IJMPD, 15, 1163

Garcia-Berro, E., et al. 2007, A\&ARv, 14, 113

Garcia-Berro, E., et al. 2011, JCAP, 05, 21

Gaztañaga, E., et al. 2002, PhRvD, 65, 023506
Guenther, B., Krauss, L., \& Demarque, P. 1998, ApJ, 498, 871

Hinshaw, G., et al. 2013, ApJS, 208, 19

Kaspi, V., Taylor, J., \& Ryba, M. 1994, ApJ 428, 713

Li, Y.-C., et al. 2013, PhRvD, 88, 084053

Mortonson, M., et al. 2014, arXiv, 1401.0046

Moss, A., et al. 2010, http://arxiv.org/abs/1004.2066

Mould, J. 2011, PASP, 123, 1030

Muller, J., \& Biskupek, L. 2007, Class. Quant. Grav., 24, 4533

Narimani, A., et al. 2012, Ap\&SS, 341, 617

Rapetti, D., et al. 2013, MNRAS, 432, 973

Riess, A., et al. 2012, ApJ, 752, 76

Solà, J. 2013, JPhCS, 453, 2015

Suzuki, N., et al. 2012, ApJ, 746, 85

Thorsett, S. 1996, PhRvL, 77, 1432

Tomaschitz, R. 2010, Ap\&SS, 325, 259

Uzan, J.-P. 2003, RvMP, 75, 403

Vandenberg, D. 1977, MNRAS, 181, 695

Verbiest, J., et al. 2008, ApJ, 679, 675

Weinberg, D., et al. 2013, PhR, 530, 87

Williams, J., \& Dickey, J. 2002, http://ilrs.gsfc.nasa.gov/docs/ williams_lw13.pdf 